
\title{
28 Research Suare \\ Halogen Free Flame-Retardant Glass Fiber Reinforced PA 66 / PPO Alloys
}

\author{
Zhenya Zhang \\ Henan Academy of Sciences Co., Ltd \\ Kunpeng Cai \\ Zhengzhou University \\ Yaxin Guo \\ Henan Academy of Sciences Co., Ltd \\ Xiaohua Liu \\ Henan University of Technology \\ Suqin He \\ Zhengzhou University \\ Hao Liu \\ Zhengzhou University \\ Miaoming Huang \\ Zhengzhou University \\ Mingcheng Yang \\ Henan Academy of Sciences Co., Ltd \\ Wentao Liu ( $\nabla$ wtliu@zzu.edu.cn ) \\ Zhengzhou University
}

\section{Research Article}

Keywords: polyamid 66, polyphenylene oxide, halogen-free, flame-retardant, glass fiber reinforced

Posted Date: June 30th, 2021

DOl: https://doi.org/10.21203/rs.3.rs-653883/v1

License: @ (i) This work is licensed under a Creative Commons Attribution 4.0 International License.

Read Full License 


\section{Abstract}

Halogen-free flame retardants are ideal plastic additives that meet carbon neutral requirements. In this work, halogen free flame retardant with glass fiber reinforced PA66/PPO composites were prepared by using coated red phosphorus (FRM-150B) and phosphorus-silicon flame retardant (WR6002). The mechanical properties, heat resistance, friction and wear properties and flame retardancy were carried out to evaluate the performances of composites using as structural parts that require heat resistance, dimensional stability and accuracy. It was found that the friction coefficient and wear volume of the composites were improved with the contents of glass fiber increased, as a result, PA66/PPO composites was obtained with excellent comprehensive performance when the content of compatilizer is $7 \%$, the glass fiber was $30 \%$, the content of FRM is $8 \%$ and the phosphorous-silicon flame retardant is $16 \%$. The flame retardant effect of FRM-150B and WR6002 in PA66/PPO was presented in the condensed phase, the results showed that the composite material with 16\% WR6002 forms a carbon layer with excellent thermal stability. On the other hand, the mechanical properties of composites were hardly affected, has important prospects in automotive components and household appliances

\section{Introduction}

Polyphenylene Oxide (PPO) also known as poly (2, 6-dimethyl-1, 4-phenylene oxide), is one of the five major engineering plastics with good thermal stability and mechanical strength. Polyphenylene ether has flame-retardant properties due to its molecular structure, and the oxygen index is $28^{[1-2]}$. The glass transition temperature $(\mathrm{Tg})$ of polyphenylene ether is $210^{\circ} \mathrm{C}$, which can maintain its good mechanical properties, flame retardant properties $\square$ and electrical properties in a large temperature range. The dielectric properties of PPO are the best among the five engineering plastics ${ }^{[3-5]}$. In addition, the dimensional stability of polyphenylene oxide is good at plastic cage, but the disadvantage of polyphenylene oxide is that it has a large melt viscosity and it is difficult to process. And the products are prone to stress cracking due to residual stress.

Nylon 66 is a thermoplastic, one of the five major engineering plastics, too, and it mostly used in electronic devices and automotive parts due to its excellent mechanical properties, outstanding molding characteristics ${ }^{[6]}$, good chemical resistance and electrical insulating features ${ }^{[7-12]}$. However, the oxygen index of nylon 66 is only 24, which is belongs to the flammable class. The flame retardancy of nylon 66 is very poor, and it will burn with the release of smoke and severe melting and dripping during the combustion process, which is the reason that greatly limits its application range ${ }^{[13-14]}$. However, nylon 66 has excellent processing fluidity due to it contains a large number of amide bonds in its main chain ${ }^{[15]}$. Moreover, with low melt viscosity. But the high-water absorption is the major disadvantage of nylon 66.

PPO and PA66 has a similar melting point. The high melt viscosity of PPO could be deduced by incorporating PA66. However, the mechanical properties of the simple blend need to be improved due to the poor interface compatibility between PA66 and PPO. Therefore, some compatibilizers, such as high 
impact polystyrene and styrene-butene copolymer, were introduced to enhance the compatibility of the PPO/PA66 ${ }^{[2-3,5]}$.

Although pure polyphenylene oxide will extinguish by itself within a period of time after ignition. However, the flame-retardant performance of the materials would be greatly reduced. after blended with PA66. It is necessary to perform flame retardant modification treatment on composites ${ }^{[7]}$. In order to meet the market's requirements for flame retardant performance in electronic devices and other aspects. Though halogen-based flame retardants still occupy the main market, its threat on environment and human health has attracted more and more people's attention.

As the country pays more and more attention to environmental protection, halogen-free flame retardants such as phosphorous flame retardants, nitrogen flame retardants etc. rise in response to the proper time and conditions ${ }^{[10-14,16]}$.

In this study, the coated red phosphorus and phosphorus-silicon flame retardants were blended into PA66/PPO, respectively. The glass fibers were added during the extrusion of the composites to strengthened the mechanical properties. Then, a halogen-free flame-retardant glass fiber reinforced PA66/PPO with excellent comprehensive performance was prepared ${ }^{[18-29]}$. The oxygen index test, vertical combustion test, friction and wear performance, mechanical properties and thermal properties were carried out to appraise effect of blending.

\section{Experimental}

\subsection{Materials and chemicals}

PPO (polyphenylene oxide) was provided by Nantong Xingchen Synthetic Materials Co., Ltd. China. Polyamide66 (PA66) was purchased by Ping-dingshan Shenma Engineering Plastics Co., Ltd. China. High impact polystyrene (HIPS) was supplied by Shanghai SECCO Petrochemical Co., Ltd. Maleic anhydride was bought by Tianjin Komiou Co., Ltd. Non-alkali non-twisted glass fiber provided by Jushi Group Co., Ltd. The coated red phosphorus (FRM-150B) and Phosphorus-silicon flame retardant (WR6002) was provided by Zibo Wanrong Chemical Co., Ltd. HIPS-g-MAH was made by the laboratory.

\subsection{Synthesis of HIPS-g-MAH}

The HIPS resin was dried in a vacuum drying oven at $95^{\circ} \mathrm{C}$ for 12 hours, and then the HIPS resin and maleic anhydride were melt-blended in an internal mixer at $180^{\circ} \mathrm{C}$ for 8 minutes at a speed of $20 \mathrm{r} / \mathrm{min}$. Finally, the melt-blended polymer was irradiated with a dose of $10 \mathrm{kGy}$ in the gamma rays emitted by the co source.

\subsection{Preparation of PA66/PPO composite}

PA66 and PPO were dried under a vacuum oven at $110^{\circ} \mathrm{C}$ for 4 hours prior to extrusion. The required additives and flame retardant materials were uniformly mixed in different proportions. And the additives, 
PA66 and PPO were extruded through a Giant SHJ-36 twin-screw extruder and pelletized. The pellets were dried in a vacuum oven at $120^{\circ} \mathrm{C}$ for 4 hours and then injection molded into samples with an injection molding machine (PL860/260, Wuxi Haitian Machinery Co., Ltd.). The detailed processing conditions for the extrusion and injection molding are listed in Table 1.

Table $1 \mathrm{~A}$

Processing conditions

\begin{tabular}{|lllllllllll|}
\hline Composition & \multicolumn{8}{|c|}{ Barrel temperature $\left({ }^{\circ} \mathrm{C}\right)$} \\
\hline PA66/PPO & GF & 1 & 2 & 3 & 4 & 5 & 6 & 7 & 8 & Export \\
$75 / 20$ & 0 & 245 & 255 & 260 & 260 & 265 & 265 & 270 & 270 & 265 \\
& $30 \%$ & 275 & 275 & 270 & 270 & 265 & 265 & 265 & 265 & 265 \\
\hline
\end{tabular}

Table 1 B

Injection conditions

\begin{tabular}{|lllllll|}
\hline Composition & \multicolumn{5}{|c|}{ Barrel temperature $\left({ }^{\circ} \mathrm{C}\right)$} \\
\hline PA66/PPO & GF & 1 & 2 & 3 & 4 & 5 \\
$75 / 20$ & 0 & 257 & 262 & 265 & 260 & 255 \\
& $30 \%$ & 267 & 272 & 275 & 270 & 265 \\
\hline
\end{tabular}

\subsection{Characterization}

The limit oxygen index (LOI) values were measured by a JF-3 digital oxygen index meter (Nanjing Jionglei Instrument Equipment Co., Ltd.) with sheet dimensions of $80 \mathrm{~mm} \times 10 \mathrm{~mm} \times 4 \mathrm{~mm}$ according to GBT 2406.1-2008. The UL-94 vertical burning level was tested on a 5402 horizontal vertical burning analyzer (Suzhou, China) according to GB/T $2408-2008$ and the dimensions of all samples were $130 \mathrm{~mm} \times 13$ $\mathrm{mm} \times 3 \mathrm{~mm}$. The tensile strength and bending strength were conducted on an electronic universal test system according to GB/T 1040-92using the (RGM-3010, Shenzhen Rigel Instrument Co., Ltd.) with the spline size was $80 \mathrm{~mm} \times 10 \mathrm{~mm} \times 4 \mathrm{~mm}$. The tensile strength test rate was $20 \mathrm{~mm} / \mathrm{min}$, and the bending strength test rate was $2 \mathrm{~mm} / \mathrm{min}$ with the test span was $64 \mathrm{~mm}$. The notched impact strength is tested by the izod impact tester according to the standard of GB/T 1843-2008, the spline size was $80 \mathrm{~mm} \times 10$ $\mathrm{mm} \times 4 \mathrm{~mm}$, the notch shape is a V-shaped mouth of $45^{\circ}$, There are five patterns in each group, and the average value is calculated. The morphology of the fracture surface obtained after broken by izod impact tester were observed by scanning electron microscopy (SEM, S-4800 Japan) at an accelerating voltage of $15 \mathrm{kV}$. The surface of samples was sputter-coated with gold before examination. The friction coefficient and wear rate were conducted on a High-speed reciprocating friction and wear testing machine according 
to GB/T3960-2016 using the (MGW02, Lanzhou Zhongke Kaihua Technology Development Co., Ltd.) with the spline size was $80 \mathrm{~mm} \times 10 \mathrm{~mm} \times 4 \mathrm{~mm}$. And the load of the sample is $10 \mathrm{~N}$, the running speed was $500 \mathrm{rpm}$, the reciprocating friction time was $1 \mathrm{~h}$, the wear radius was $3 \mathrm{~mm}$. The thermogravimetric analysis (TGA) was carried out on the STA-409 PC synchronous thermal analyzer (NETZSCA, Germany) using a heating rate of $10^{\circ} \mathrm{C} / \mathrm{min}$ in nitrogen atmosphere. The heat distortion temperature (HDT) was tested according to GB/T 1642-2004 by Thermal Deformation Vicat Softening Temperature Tester (HDT/V1002, Chengde), the load was $1.80 \mathrm{MPa}$, the heating rate was $2^{\circ} \mathrm{C} / \mathrm{min}$.

\section{Results And Discussion}

\subsection{Flame retardancy of PA66/PPO/FRM blends}

Although numerous tests have been developed to examine the flame retardant properties of blends, the vertical burning test (UL-94) and LOI test are the most commonly used methods for testing the flame retardant properties of polymer materials. The limit oxygen index (LOI) and vertical burning testing (UL94) were carried out to investigate the flame retardancy of the PA66/PPO/FRM blends. The corresponding results were listed in Table 2 and Table 3, respectively. Obviously, the PA66/PPO composites was highly flammable with a low LOI value (24 vol.\%) and no rating at the UL-94 testing accompanied by severe melt dripping. It can be seen from Table 2 that the oxygen index of the material reached $39.5 \%$ when 10 parts of coated red phosphorus added, and the flame retardant passed V-0 ratting. Because the appropriate red phosphorus was added to the composites, a membrane will be formed in the carbon layer to prevent external oxygen and some combustibles when the sample was burned, thereby an effective preventing combustion can be achieved. However, the limit oxygen index of the composites was decreased with more FRM added. This phenomenon maybe caused by the fact that red phosphorus itself was a kind of flammable material. it would release a lot of heat during combustion when too much red phosphorus added, which will make the material easy to burn, and decrease the flame retardancy of the composites.

WR6002 is a commercialized flame retardant that contains both phosphorus and silicon. Phosphorus and silicon can form a denser carbon layer during combustion, thereby achieving better effects in flame retardant. Phosphorus-based flame retardants and silicon-based flame retardants are non-toxic green flame retardants with good smoke suppression effects. They can form an expanded carbon layer during combustion to achieve heat insulation and oxygen isolation to prevent the burning of materials. Table 2, 3 and the Fig. 1 were the results of the flame retardant properties of PA66/PPO/30\% GF with different content of phosphor-silicon flame retardants. The analysis of the table showed that as the number of phosphor-silicon flame retardants increases, the limiting oxygen index of the composite material gradually increase. When the glass fiber content was $30 \%$ and the flame retardant content is $20 \%$, the limiting oxygen index of the material reaches the maximum $35.7 \%$, and the flame retardant grade was $\mathrm{V}$ 0 , which meets the flame retardant standard. However, in conjunction with the analysis of the mechanical properties of the material in Fig. 5 , the overall performance of the materials is optimal when $30 \%$ glass fiber is added and the content of the phosphor-silicon flame retardant is $16 \%$. 
Table 2

LOI and UL-94 test results of the PA66/PPO/FRM-

$150 \mathrm{~B}$ blends

\begin{tabular}{|lll|}
\hline Sample & LOI (\%) & UL-94 \\
\hline PA66/PPO/30\%GF & 25 & No \\
\hline PA66/PPO/30\%GF/8\%FRM & 40.29 & V-1 \\
\hline PA66/PPO/30\%GF/10\%FRM & 39.488 & V-0 \\
\hline PA66/PPO/30\%GF/12\%FRM & 38.272 & V-0 \\
\hline PA66/PPO/30\%GF/14\%FRM & 36.65 & V-0 \\
\hline
\end{tabular}

Table 3

$\mathrm{LOI}$ and UL-94 test results of the PA66/PPO/WR6002 blends

\begin{tabular}{|lll|}
\hline Sample & LOI (\%) & UL-94 \\
\hline PA66/PPO/30\%GF & 25 & No \\
\hline PA66/PPO/30\%GF/4\%P-Si & 31 & V-2 \\
\hline PA66/PPO/30\%GF/8\% P-Si & 33 & V-1 \\
\hline PA66/PPO/30\%GF/12\% P-Si & 33.6 & V-1 \\
\hline PA66/PPO/30\%GF/16\% P-Si & 34.6 & V-0 \\
\hline PA66/PPO/30\%GF/20\% P-Si & 35.7 & V-0 \\
\hline
\end{tabular}

\subsection{The influence of compatibilizer on the properties of PA66/PPO composite}

Compared with the query data, it was found that MAH has the characteristic peak of $\mathrm{C}=\mathrm{O}$ of cyclic anhydride at about $1850 \mathrm{~cm}^{-1}$ and $1780 \mathrm{~cm}^{-1}$, and the characteristic peak of carboxycarbonyl $(\mathrm{C}=0)$ after hydrolysis of cyclic anhydride at about $1700 \mathrm{~cm}^{-1}$. The graft compatibilizer HIPS-g-MAH has corresponding characteristic peaks of maleic anhydride in corresponding places, indicated that the graft compatibilizer has the characteristic peak of maleic anhydride, indicating that HIPS and MAH have successfully undergone the grafting reaction. Compared with pure HIPS, the purified reaction blend has three more characteristic peaks, and these peaks were just the characteristic peaks of maleic anhydride, indicated that the high impact polystyrene was grafted with maleic anhydride. The above infrared spectra prove that the grafting reaction between high impact polystyrene and maleic anhydride has formed the graft HIPS-g-MAH. 
The previous work verified the influence of different contents of compatibilizer on the tensile strength and impact strength of the matrix material. The experiment shows that with the increase of the compatibilizer content, the tensile strength of the PA66/PPO composite material first increases and then decreases.

When the content of the compatibilizer is $7 \%$, the tensile strength of the material reaches $71.95 \mathrm{MPa}$. This is because HIPS-g-MAH is used as a compatibilizer. Maleic anhydride is a functional monomer. It will react with the terminal amine groups of PA66 to form HIPS-g-PA66 at the two-phase interface, which makes the interface layer thicker, reduces the interfacial tension and improves the adhesion. However, because the added amount of the compatibilizer is too high, unreacted compatibilizer may accumulate in the interface layer, which will affect the tensile properties of the material. The notched impact strength of the composite material increases with the increase of the compatibilizer content.

The measurement of thermal deformation temperature (HDT) and the measurement of tensile strength and impact strength were carried out to study the thermal and mechanical properties of PA66 and FRPA66. It can be seen from Fig. 2 that the tensile strength and impact strength of composites have been greatly improved with the increase of glass fiber content. When the glass fiber content is $30 \%$, the impact strength of the composites reaches the maximum and the performance is optimal. It was obvious that the blending of glass fiber in the PA66/PPO matrix not only improved the bonding force of the interface, but the physical properties of the composites were improved. The matrix material is firmly bonded to the matrix after being blended with the glass fiber, and the material will preferentially transfer the load to the glass fiber when it was loaded. The interface plays the role of transferring stress and gradually spreading the load to the entire composites, greatly improved the load bearing capacity, and the size has become relatively stable.

It can be found from Fig. 3 that the thermal deformation temperature of the composites was $70^{\circ} \mathrm{C}$ when the glass fiber content was 0 . With the addition of glass fiber, the heat distortion temperature of composites has been greatly improved, and with the increase of glass fiber content, the heat distortion temperature of composites has been continuously improved. The reason was that the glass fiber itself has high heat resistance, and after blending with the matrix material, it increases the cohesive force of the components of the composites. Moreover, the glass fiber plays a role in the heterogeneous nucleation of the composites, which allows the molecular chains of the matrix to be arranged in an orderly manner within a short distance, and the movement was hindered, so more energy was required to make the polymer chains slip and movement. Therefore, the overall heat resistance of the material was improved, and the heat distortion temperature is also greatly increased.

\subsection{Friction and Wear Performance}

Polymers are more prone to adhesive wear when subjected to friction. Figure 4 shows the friction coefficient graph and the analysis graph of the wear rate of composites with different glass fiber content. It can be seen from Fig. 3 that with the glass fiber content increased from 0 to $10 \%$, the friction coefficient of the composites decreased from 0.47 to 0.34 , and the friction coefficient has been improved with the glass fiber content increased. The friction coefficient is 0.3 , and the wear rate also drops from $14.4-1.1 \%$ when the glass fiber content is $30 \%$. This is because glass fiber is a kind of wear-resistant material. 


\subsection{Mechanical properties of PA66/PPO/GF/FRM}

The mechanical properties of microcapsule-coated red phosphorus applied to PA66/PPO composites were shown in Fig. 5. It can be seen that after only adding coated red phosphorus, the tensile strength of the composites did not change significantly with the increase of the coated red phosphorus content. However, the impact strength of the material began to decrease as the content of coated red phosphorus increased. Because after coated red phosphorus added to the composites, these red phosphorus particles were likely to become stress concentration points, which weaken the resistance of the material when subjected to external forces. With the content of coated red phosphorus increased, the stress concentration point also increases, resulting in a gradual decrease in impact performance. After $30 \%$ glass fiber added, the mechanical properties of the flame-retardant composites have been significantly improved. When the content of coated red phosphorus is $8 \%$, the impact strength of the composites without glass fiber was $5.8 \mathrm{~kJ} / \mathrm{m}^{2}$, and the impact strength of glass fiber reinforced red phosphorus flame retardant material reached $7.08 \mathrm{~kJ} / \mathrm{m}^{2}$, increased $22 \%$, a composite with excellent comprehensive mechanical properties was obtained.

Figure 6. Tensile properties (left picture) and impact properties (right picture) of composites with different phosphorus-silicon (WR6002) flame retardant content.

\subsection{TGA and DTG of blends}

In an inert atmosphere (nitrogen), the thermal decomposition processes on the TGA curves displayed for PA66/PPO, PA66/PPO/GF, PA66/PPO/GF/P-Si, and PA66/PPO/GF/FR composites in Fig. 7. It can be seen from the figure that although the mechanical properties of the material enhanced after the glass fiber added. However, due to the "candle heart effect" of glass fiber, the weight loss of the material was increased compared to PA66/PPO. The introduction of WR6002 and FRM into the PA66/PPO/GF was found to reduce the temperature of the onset decomposition temperature of PA66/PPO/GF. The weight loss of the material increased from $28.8-52 \%$ after adding phosphorous and silicon flame retardant. The $\mathrm{T}_{\max }$ of the PA66/PPO/GF was $472^{\circ} \mathrm{C}$, but after WR6002 added, the $\mathrm{T}_{\max }$ was improved to $504^{\circ} \mathrm{C}$. With the addition of flame retardants, the more stable and higher quality carbon layer was formed. It can be seen from the curve that the carbon residue rate of the material also improved with the increase of the flame retardant addition. This is because with the addition of the coated red phosphorus flame retardant and phosphorus silicon flame retardant, the material can form polyphosphate during combustion, which plays a role in blocking oxygen and heat transfer. WR6002 is a phosphorous silicon flame retardant. Due to the joint action of $\mathrm{Si}-\mathrm{O}-\mathrm{Si}$ and phosphorous elements, the isolation layer formed was more stable than red phosphorous, which achieved a better flame retardant effect.

\subsection{Fracture appearance of blends}

SEM images of notch impact surface of different samples after test were presented in Fig. 8. It can be seen from $A$ and $B$ that the particle size distribution between the two dispersed phases before adding the compatibilizer was small, and the distribution was relatively clear, the parabolic dimple phenomenon was 
more obvious, and the two phases penetrate the uniform sea-island structure. With the addition of the compatibilizer, we can clearly see that the phase morphology of the blends is improved. The phase interface of the blends becomes blurred, which reduced the interfacial tension and refined the dispersed phase, resulting in enhanced material compatibility. The broken surface presents a co-continuous phase structure, and the thicker the two-phase interface layer, the more obvious the compatibilization effect, which is consistent with the trend of increasing mechanical properties. The $\mathrm{C}$ is the composites with glass fiber. From the $D$ and $E$, it can be seen that a clear cavity structure after the glass fiber was broken. After the glass fiber was pulled out, it can be clearly seen that the adhesive materials were taken out, indicating that the glass fiber has improved the phase of the composites. Capacitance improves the mechanical properties of composites. After the flame retardant added, the morphology of the fracture site has changed significantly. It can be seen from Fig. $8 \mathrm{C}$ that the fracture surface of the composites after adding the flame retardant was bark-like, which will damage the mechanical properties of the blends.

Based on the above results, a flame-retardant mode of action was proposed (Fig. 9). The Si-O-Si will promote the degree of carbonization of flame-retardant materials in providing good flame retardancy of PA66/PPO blends. The cooperative of Si and P enhanced the stability of char layer, contributed to the phosphorus and silicon-rich cross-linked char, which play the flame retardant effect in condensed phase. In addition, released incombustible gases during the combustion, including ammonia and steam, could dilute the oxygen concentration and took the heat away, contributing to the gas-phase mode of action. Therefore, FRM exert the flame retardant effect both in gas phase and condensed phase.

\section{Conclusions}

In summary, by adding irradiated HIPS-g-MAH and glass fiber to PA66/PPO, the composite material was compatibilized and modified by glass fiber, and PA66/PPO was reinforced and modified. The composite material was modified by adding flame retardant (IFR) FRM-15B and WR6002 respectively. The flame retardant efficiency and mechanical properties of PA66/PPO have been significantly improved. Different contents of glass fiber and compatibilizer have a significant impact on the mechanical properties of PA66/PPO composites. According to the experimental data, when the compatibilizer content is $7 \%$ and the glass fiber content is $30 \%$, the composite material can obtain the greatest interfacial adhesion and the best mechanical properties. As the content of red phosphorus coated in the composite material increases, the oxygen index of the composite material first increases and then decreases. When FRM-15B is added to the composite material, a glass-like isolation layer of metaphosphoric acid is formed on the surface of the material during combustion to block the combustion. When the content of FRM-15B is $10 \%$, the flame retardant performance reaches the $\mathrm{V} 0$ level, the flame retardant performance is the best, and the impact on the mechanical properties of the composite material is minimal. After research, it is found that the oxygen index of the spline after adding glass fiber is higher than that of the spline without glass fiber, which proves that glass fiber can increase the flame retardant performance of the material while enhancing the mechanical properties of the material. Experiments show that the flame retardant ability of 
phosphorous silicon flame retardant is weaker than that of coated red phosphorus. However, when the phosphorus-silicon flame retardant content is $16 \%$, the composite material can also be extinguished in a short time. The flame retardant performance of the composite material after adding $16 \%$ phosphorous silicon flame retardant reaches the $\mathrm{V} 0$ level, and the carbonization after combustion and the isolation layer on the surface of the material are better than the blended material with red phosphorus. In addition, the overall performance of composite materials is better than that of adding coated red phosphorous materials.

\section{Declarations}

\section{Acknowledgement:}

The authors would like to acknowledge the research platform of the Institute of Isotope, Henan Academy of Sciences Co., Ltd., No.7 Songshan South Road, Zhengzhou 450015, China.

\section{Funding:}

This research was funded by Henan Academy of Sciences, Grant Number "190404005", and Jiaozuo innovation and entrepreneurship leading team project (2020TD005).

\section{Conflicts of Interest:}

The authors declare no conflict of interest.

\section{References}

1. Wang, J., Tsou, A. H., Passino, H. L. \& Favis, B. D. PPE-g-HDPE in high-performance poly(pphenyleneether)/ polyethylene blends: synthesis and compatibilization effects, Polymerpp. 92102(The International Journal for the Science and Technology of Polymers, 2018). 11

2. Wu, S. J., Lin, T. K. \& Shyu, S. S. Cure behavior, morphology, and mechanical properties of the melt blends of epoxy with polyphenylene oxide. Journal of Applied Polymer ence, 75 (1), 26-34 (2015).

3. Ran, J. et al. Remarkable enhancement of mechanical and tribological properties of polyamide 46/polyphenylene oxide alloy by polyurethane-coated carbon fiber,High Performance Polymers, 2019.1122-1131.

4. Hyun, K. et al. Reactively formed block and graft copolymers as compatibilizers for polyamide 66/PS blends,Polymer, 2004.197-206.

5. Hadimani, P., Murthy, H. N. N. \& Mudbidre, R. Processing and Characterization of Polyphenylene Ether/Polystyrene/Nylon-6 Ternary Blends. International Polymer Processing Journal of the Polymer 
Processing Society, 35 (2), 169-183 (2020).

6. Ruixiang, Y. et al. Preparation and Properties of PPE-g-MAH/PA66 Blends,Engineering Plastics Application, 2016.41-45.

7. Tol, R. T. et al. Phase morphology and stability of co-continuous (PPE/PS)/PA6 and PS/PA6 blends: effect of rheology and reactive compatibilization,Polymer, 2004.2587-2601

8. Jana, S. C., Patel, N. \& Dharaiya, D. Compatibilization of PBT-PPE blends using low molecular weight epoxy., 42 (21), 8681-8693 (2001).

9. Chiang, C. R. \& Chang, F. C. Polymer blends of polyamide- 6 and poly(phenylene oxide) compatibilized by styrene-co-glycidyl methacrylate. Journal of Applied Polymer ence, 61 (13), 2411-2421 (1996).

10. Gu, L., Chen, G. \& Yao, Y. Two novel phosphorus-nitrogen-containing halogen-free flame retardants of high performance for epoxy resin. Polymer Degradation \& Stability, 108 (oct.), 68-75 (2014).

11. Liu, Y. et al. Flame-Retardant Effect of Sepiolite on an Intumescent Flame-Retardant Polypropylene System. Ind. Eng. Chem. Res, 50 (4), 2047-2054 (2011).

12. Pan, Y. et al. Polydimethylsiloxane wrapped aluminum diethylphosphinate for enhancing the flame retardancy of polyamide 6,Journal of Applied Polymer ence, 2020:49027.

13. Li, L., Li, B. \& Tang, F. Influence of maleic anhydride-grafted EPDM and flame retardant on interfacial interaction of glass fiber reinforced PA-66. Eur. Polymer J, 43 (6), 2604-2611 (2007).

14. Zhan, Z., Xu, M. \& Li, B. Synergistic effects of sepiolite on the flame retardant properties and thermal degradation behaviors of polyamide 66/aluminum diethylphosphinate composites. Polymer Degradation \& Stability, 117 (jul.), 66-74 (2015).

15. Jie-Hui, F. U. et al. The Compatibilization and Toughening Modification of PPO/PA Alloy,China Plastics Industry, 2014.36-39.

16. LONG, L. et al. Function of the aryl group in bis DOPO phosphonate on reducing fire hazards of polyamide 6 composites,Journal of Applied Polymer Science, 2020.137.

17. Zhu, J. et al. Preparation of graphene oxide modified glass fibers and their application in flame retardant polyamide 6,Polymers for Advanced Technologies, 2020(11).1709-1718.

18. Wit, C. A. D. An overview of brominated flame retardants in the environment., 46 (5), 583-624 (2002).

19. Watanabe, I. \& Sakai, S. I. Environmental release and behavior of brominated flame retardants. Environ. Int, 29 (6), 665-682 (2004).

20. Veen, I. V. D. \& Boer, J. D. Phosphorus flame retardants: Properties, production, environmental occurrence, toxicity and analysis., 88 (10), 1119-1153 (2012).

21. Schell, K., Rowen, J. B. \& Camarota, A. S. Flame retardant and smoke supressive additive powder for polymeric thermoplastics and thermoset resins: US 2005. Patent 6930138

22. Guo, X. et al. Great enhancement of efficiency of intumescent flame retardants by titanate coupling agent and polysiloxane. Polymers for Advanced Technologies, 32 (1), https://doi.org/10.1002/pat.5059 (2020). 
23. Kanno, T. et al. Flame Resistant Nylon6,6 Composites with Improved Mechanical Strength by the Combination of Additive and Reactive-Type Flame Retardants. Polym. J, 39 (4), 347-358 (2007).

24. Kim, N. K., Lin, R. J. T. \& Bhattacharyya, D. FIRE RETARDANT PROPERTIES OF WOOL FIBRE COMPOSITES, //22nd International Conference on Composites or Nano Engineering. 2014.

25. Rudzinski, S., Ussler, H. \& Harnisch, L. C, et al. Glass fibre reinforced polyamide composites: Thermal behaviour of sizings. Composites Part A Applied ence \& Manufacturing, 42 (2), 157-164 (2011).

26. Zhang, Y., Liu, Y. \& Wang, Q. Synergistic effect of melamine polyphosphate with macromolecular charring agent novolac in wollastonite filled PA66. Journal of Applied Polymer ence, 116 (1), 45-49 (2010).

27. Akinci, A., Sen, S. \& Sen, U. Friction and wear behavior of zirconium oxide reinforced PMMA composites. Composites Part B, 56 (jan.), 42-47 (2014).

28. Conghui, Z. et al. Research on Friction and Wear Performance of Commercially Pure Titanium Welded Joints by Ultrasonic Impact-Rolling Processing,Lubrication Engineering, 2019.25-30.

29. Jincheng, R. et al. Significant improvement of tribological performances of polyamide 46/polyphenylene oxide alloy by functionalized zirconium phosphate. Tribol. Int, 128, 204-213 (2018).

\section{Figures}




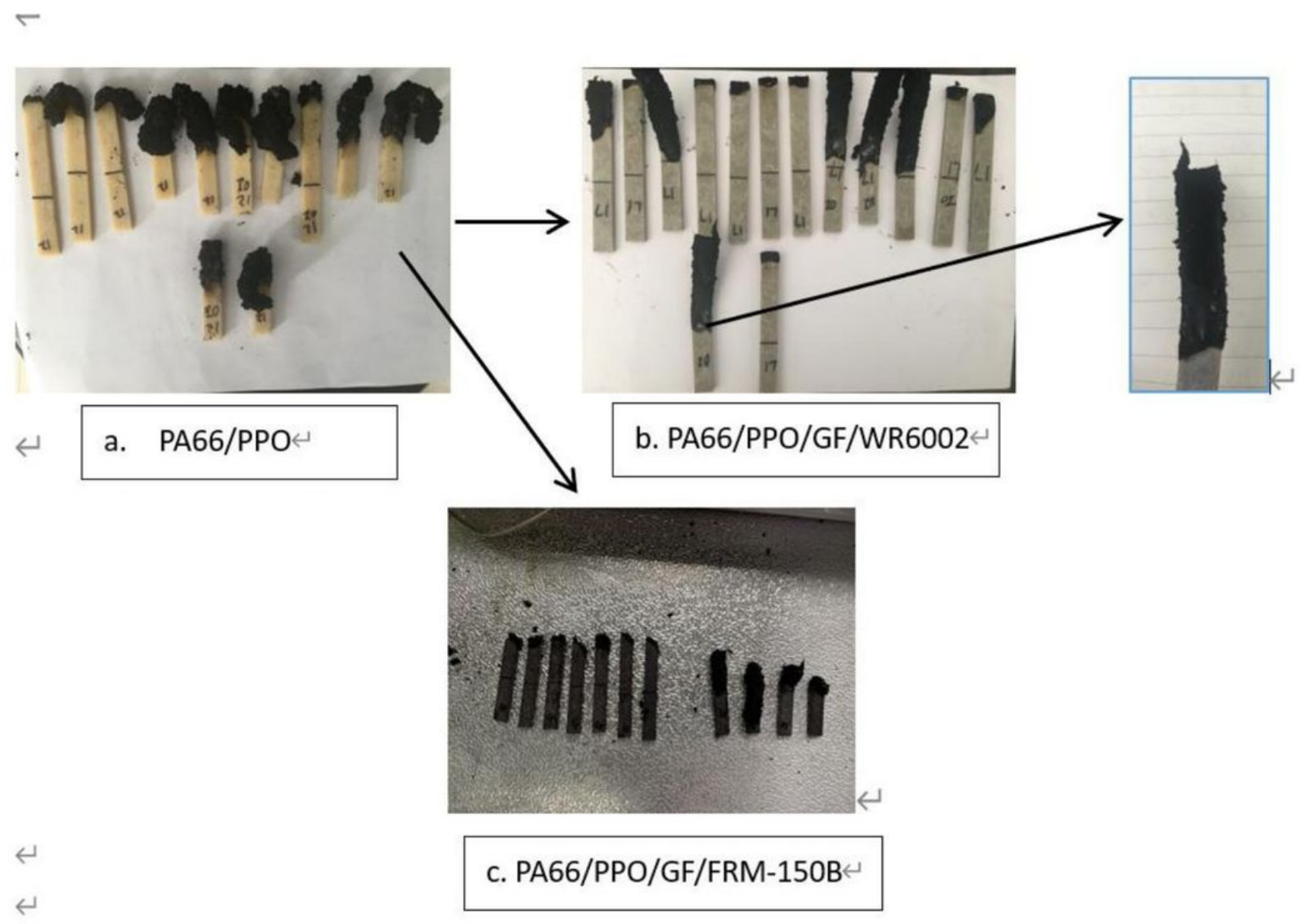

Figure 1

Photographs of different IFR samples after calcination. 


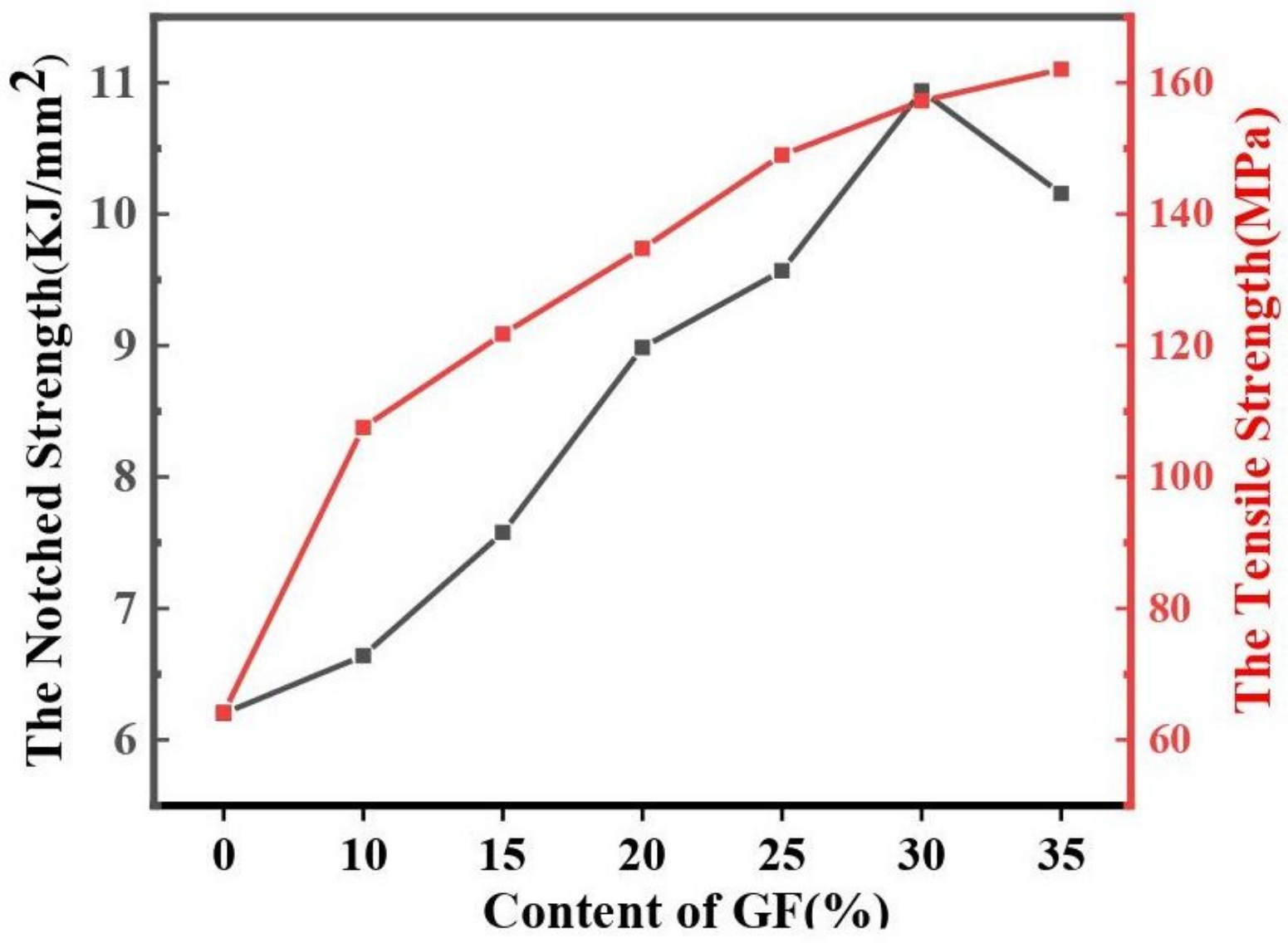

Figure 2

The influence of glass fiber content on the mechanical properties of PA66/PPO 


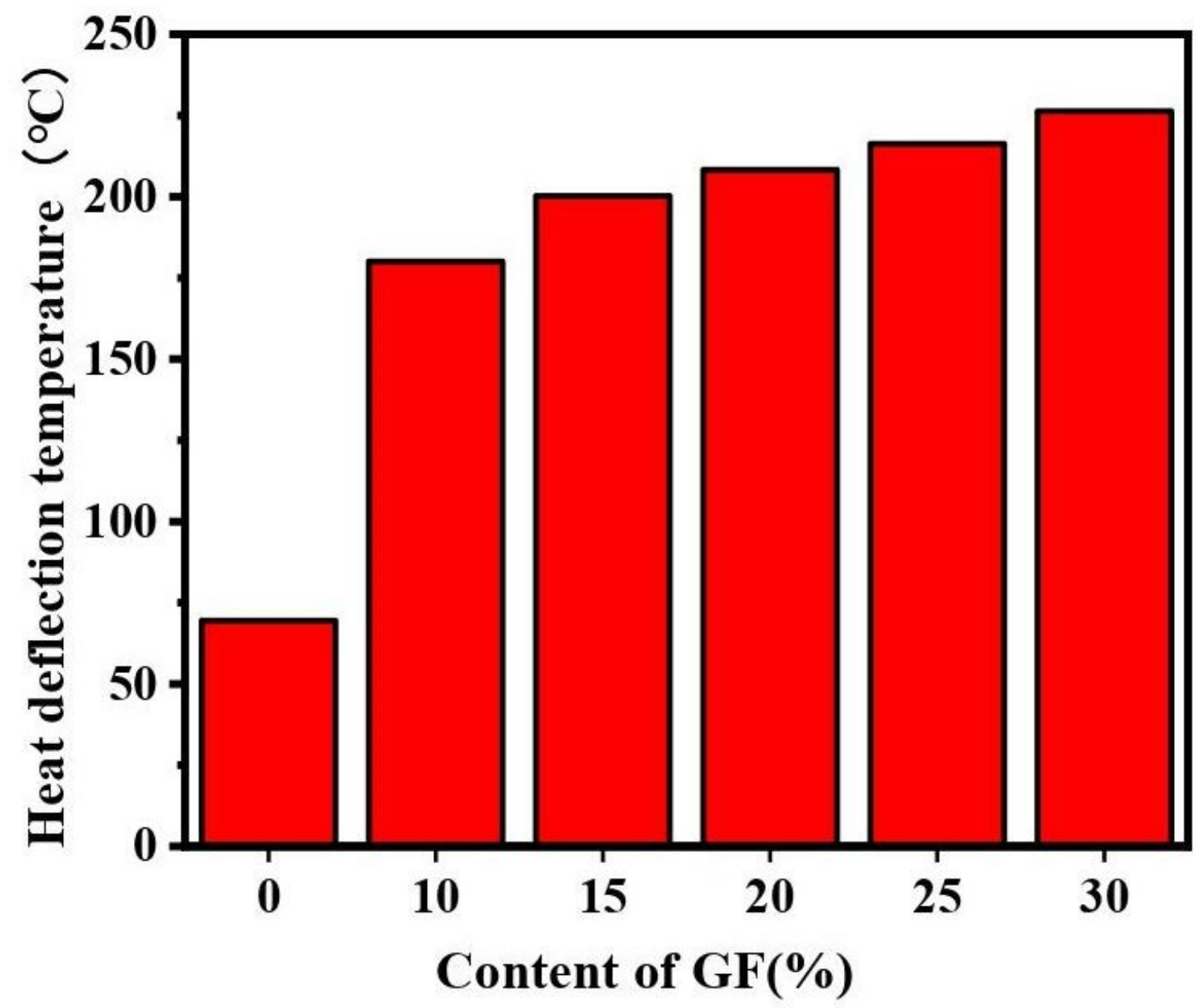

Figure 3

The influence of glass fiber content on the thermal deformation temperature of PA66/PPO 

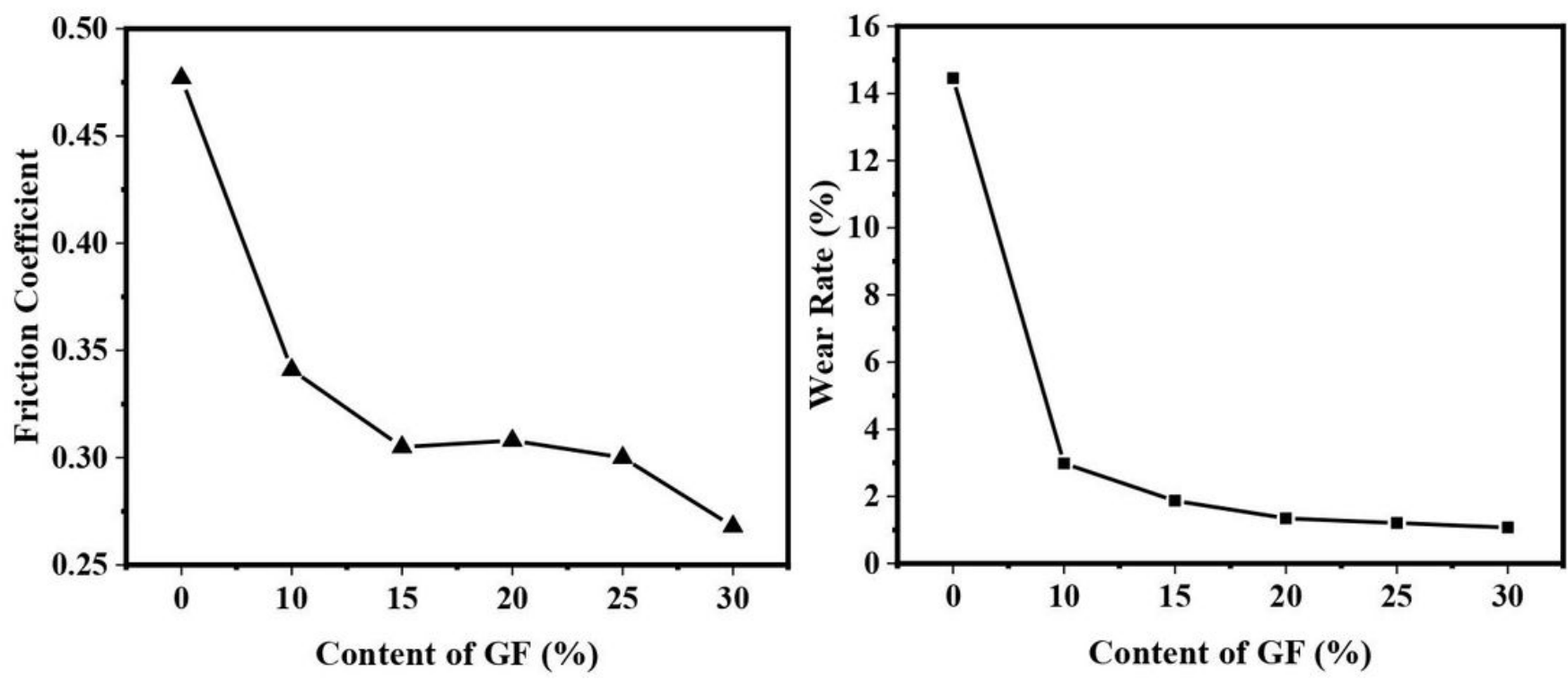

Figure 4

The effect of glass fiber content on the friction and wear properties of PA66/PPO
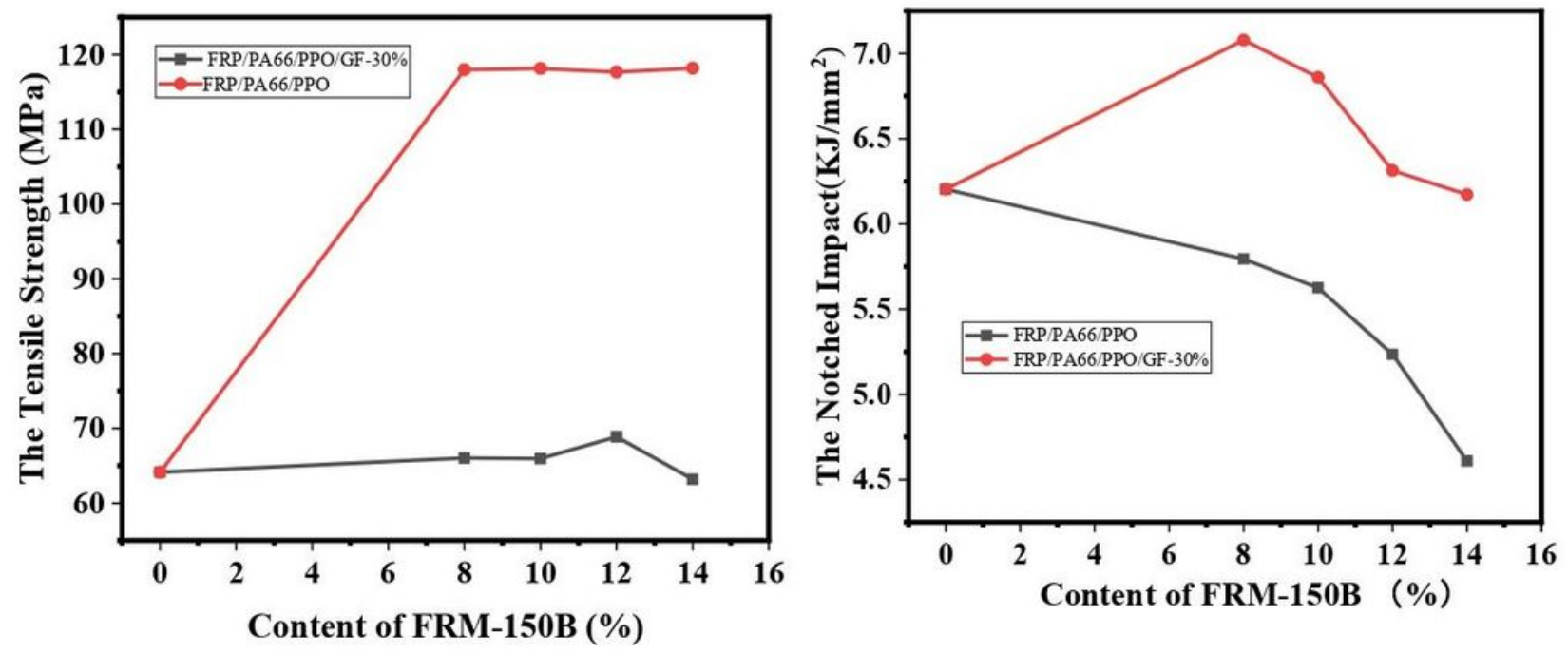

Figure 5

Tensile properties (left picture) and impact properties (right picture) of composites with different red phosphorus (FRM-150B) content 

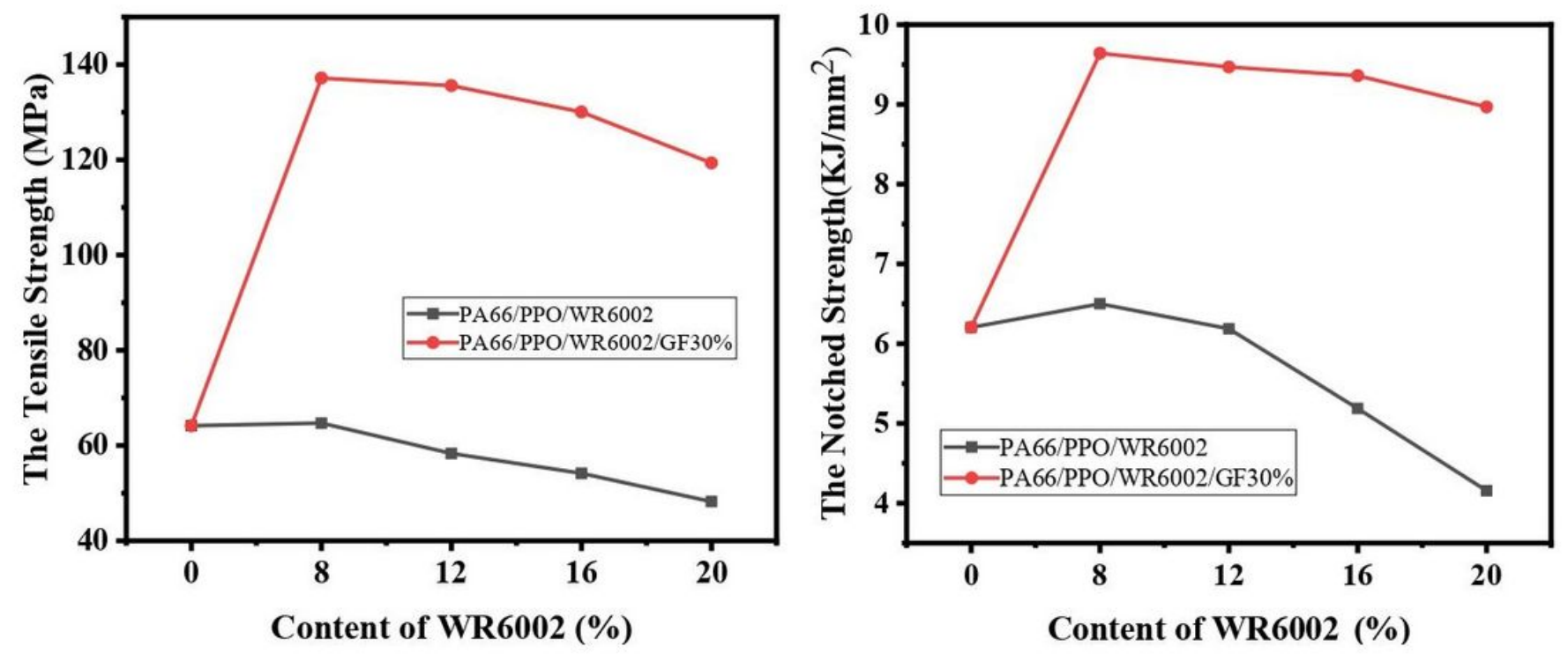

Figure 6

Tensile properties (left picture) and impact properties (right picture) of composites with different phosphorus-silicon (WR6002) flame retardant content.
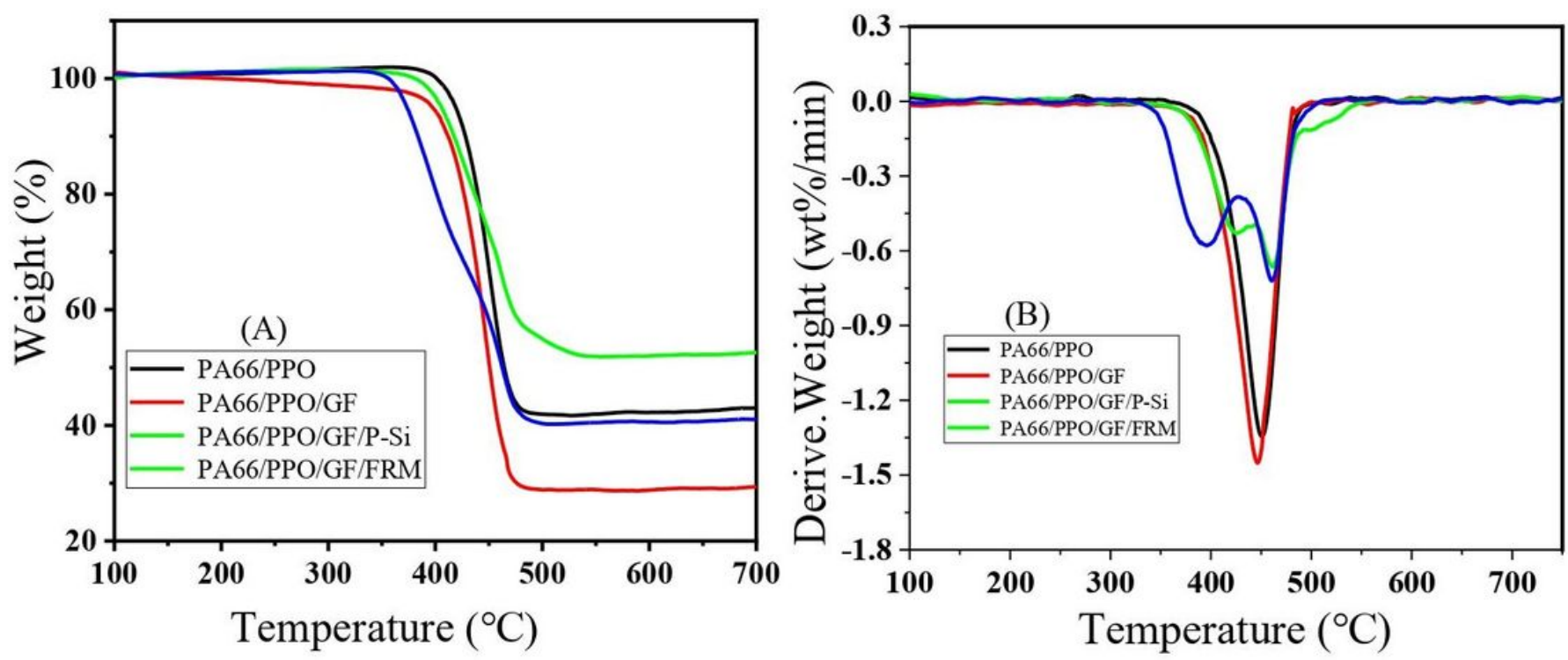

Figure 7

A, TGA and B, DTG curves of different samples. 

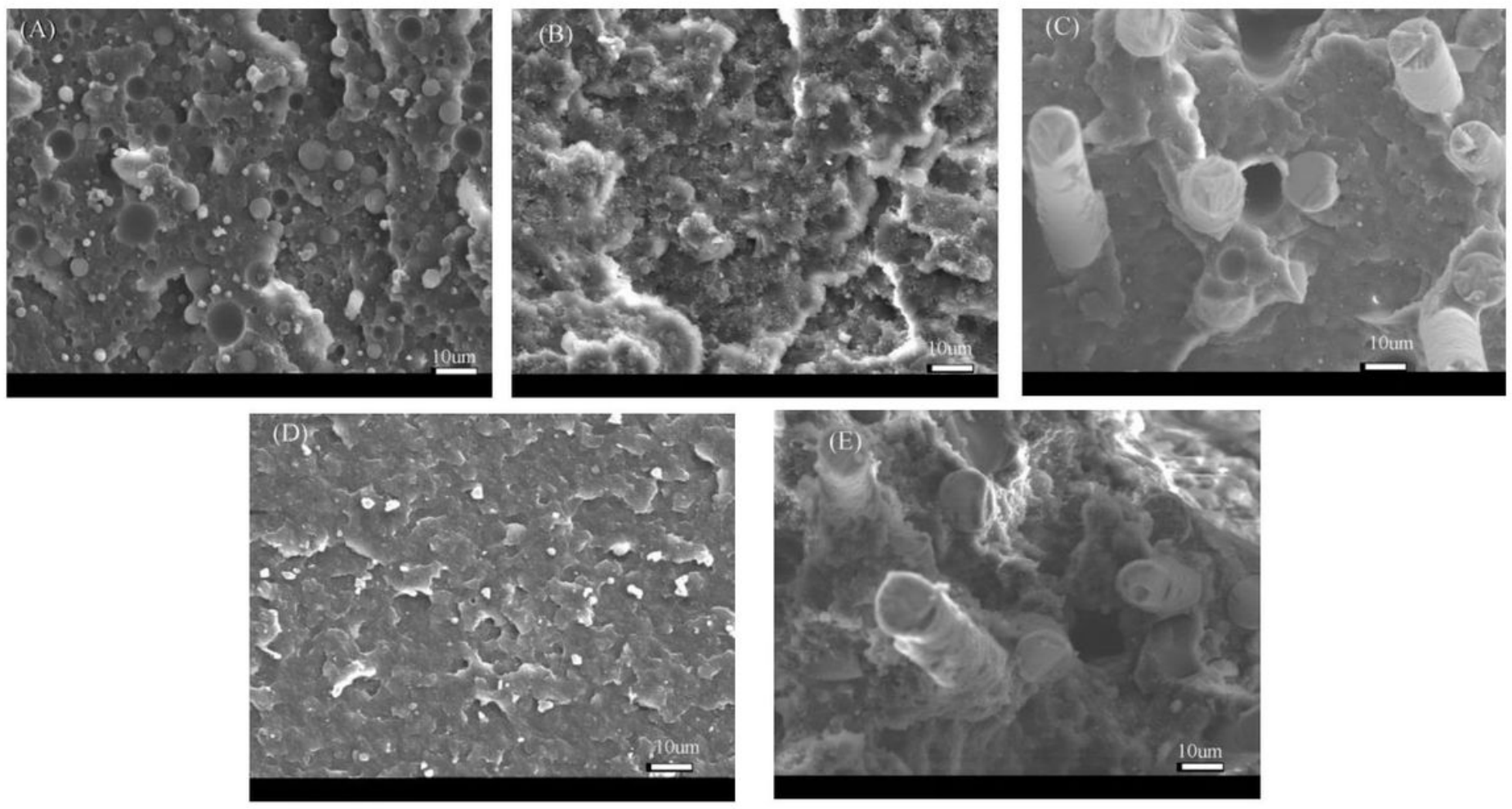

\section{Figure 8}

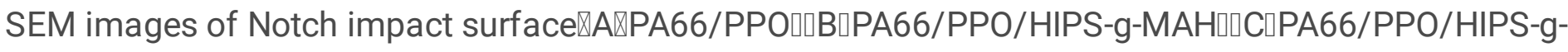
MAH/GFuDDPA66/PPO/HIPS-g-MAH/WR60020EETPA66/PPO/HIPS-g-MAH/WR6002/GF

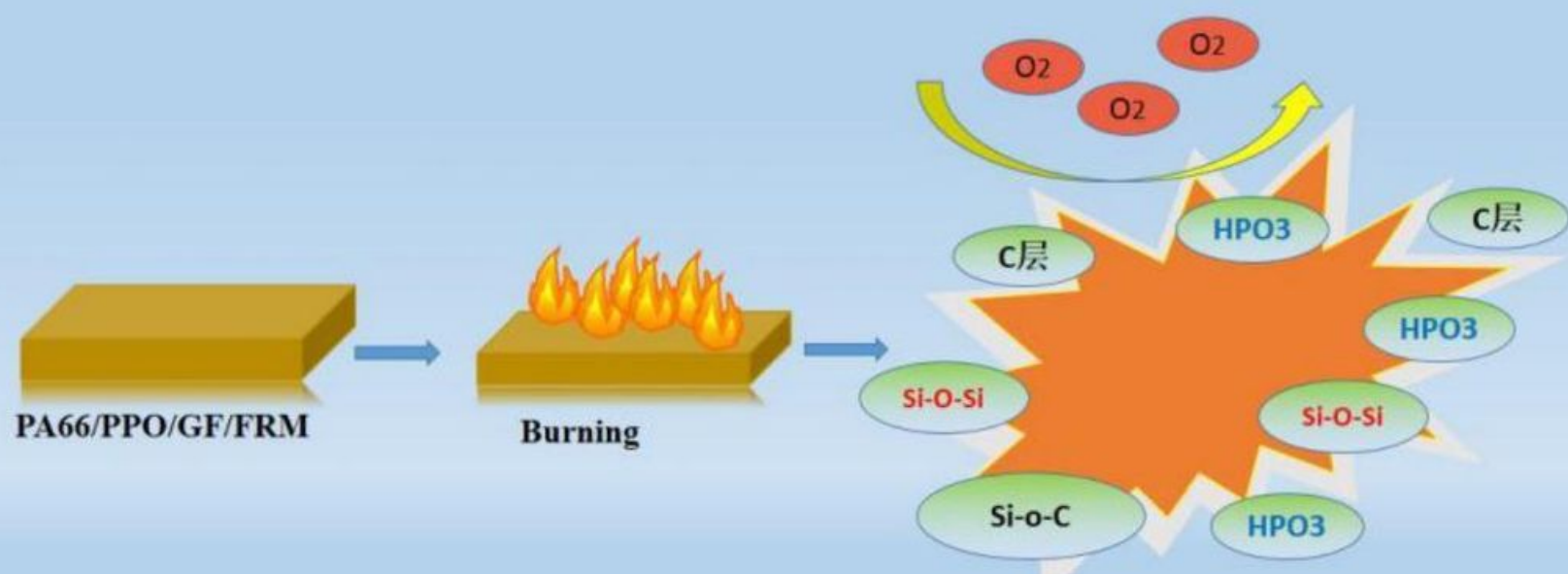




\section{Figure 9}

The flame-retardant mechanism of PA66/PPO/FR composites

\section{Supplementary Files}

This is a list of supplementary files associated with this preprint. Click to download.

- support.docx 